\title{
FIXING DEVICE AERODYNAMICS OF THE WALL CLIMBING ROBOT
}

\author{
V.G. Chashchukhin \\ Institute for Problems in Mechanics of the Russian Academy of Sciences, \\ Moscow, Russia \\ www.ipmnet.ru \\ Moscow Institute of Physics and Technology, \\ Moscow, Russia \\ www.mipt.ru \\ E-mail: ketlk@mail.ru
}

\begin{abstract}
The aerodynamics of a fixing device intended for robots of vertical movement is investigated. Fixation is carried out by creating a vacuum under the bottom of the robot through a fan. Theoretical calculations were carried out to assess the dependence of the pressing force on the flow rate of air pumped out by the fan. A comparison is also made with experimental data obtained using the created model of the fixing device.
\end{abstract}

Keywords: wall climbing robot, fixing device, aerodynamic adhesion.

\section{Related works}

Aerodynamic adhesion is often used to fixing miniature wall climbing robots. Surface fixation is carried out using centrifugal or axial fans with various shapes of ducts. In $[1,2]$ a fixing device's aerodynamics simulations of the "city climber" robot is considered. The device is based on a centrifugal fan, at the outlet of which the flow is thrown in the direction from the surface, creating an additional pressing force. Also aerodynamics simulations of fixing devices with centrifugal and axial fans are represented in [3,4]. In [5-7] using a fluid network theory is proposed for calculating the adhering characteristics of the sliding suction cup of the wall climbing robot. It is assumed that in the suction cup the flow is considered as radial. The flow radiality is also used in [8] to study the fixing device which is an impeller that rotates under the bottom of the robot. Experimental investigation of this type of a robot is given in [9]. An detailed experimental study of a fixing device using an axial fan is presented in $[10,11]$. Fixing device without impeller that uses vortex flow to generate a pressing force is consider in [12]. The problem of aerodynamic adhesion to the surface is similar in essence to the problem of holding a hovercraft above the surface, considered, for example, in [13]. In this paper investigates the dependence of the fixing force on the air flow rate created by the fan based on the Bernoulli equation. This study will theoretically evaluate the effectiveness of various types of seals of wall climbing robots with aerodynamic adhesion to the surface.

\section{Fixing device aerodynamic model}

Consider the aerodynamic fixing device of a crawling robot. Let's introduce a Cartesian coordinate system as shown at fig. 1. The robot's vacuumed chamber consists of several areas. Areas (1) under the bottom of the body of the device and areas (2) under the fan.

In the area (1), we denote the pressure as $p_{1}(x)$, air flow rate as $v_{1}(x)$, distance from the surface to the bottom of the fixing device as $h$, flow area as $S_{1}(x)=2 \pi x h,\left(r_{2} \leq x \leq r_{1}\right)$, in this area coordinate $x$ takes values from the interval $r_{2}<x<r_{1}$, where $r_{2}$ - radius of the area under the fan, $r_{1}$ - external radius of the body. Since the height of the robot is insignificant than changes in atmospheric pressure depending on $y$ is negligible. In the area 


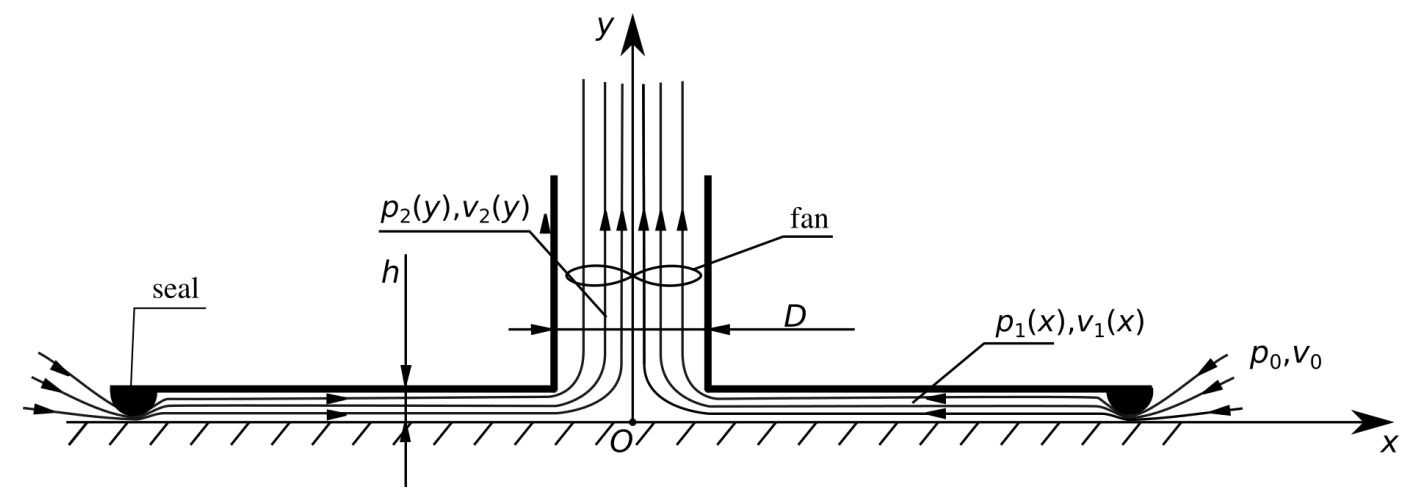

Fig. 1. Air current lines in the fixing device

(2) $0 \leq x<r_{2}$ we assume that the pressure $p_{2}$ does not depend on the coordinate $y$. The rate in this area $v_{2}$, the width of the area $2 r_{2}$, flow area $S_{2}=\pi r_{2}^{2}$. The distance from the surface to the bottom and the radius of the cross- section at the entrance to area (2) must be related by the ratio $r_{2}(y=h)=2 h$ in order to avoid cross-section differences at the junction of area (2) and area (1). Atmospheric pressure outside $p_{0}$, the flow rate under the bottom is obviously much lower than the speed of sound, so the density of air $\rho$ is constant value. We will consider the steady flow mode. Than the mass air flow rate $Q$ generated by the fan is constant and the flow rate is determined by the following expressions

$$
v_{1}=\frac{Q}{\rho S_{1}}, \quad v_{2}=\frac{Q}{\rho S_{2}}
$$

The pressure distribution in the flow is given by the Bernoulli equation

$$
p_{0}=p_{1}(x)+\frac{\rho v_{1}^{2}(x)}{2}=p_{2}+\frac{\rho v_{2}^{2}}{2}
$$

substituting (1) in (2) we obtain the following expressions

$$
p_{1}(x)=p_{0}-\frac{Q^{2}}{8 \rho \pi^{2} x^{2} h^{2}}, \quad\left(r_{2} \leq x \leq r_{1}\right) ; \quad p_{2}=p_{0}-\frac{2 Q^{2}}{\rho \pi^{2} r_{2}^{4}}
$$

Reactive pressing force due to air flow from the fan directed from the surface is representable as follows.

$$
F_{R}=\frac{\Delta m}{\Delta t} v_{2}=Q v_{2}=\frac{Q^{2}}{\pi \rho r_{2}^{2}}
$$

In the case of hovering in air, this force is a force that compensates for the weight of the robot. In the case of a centrifugal fan, this force is present if the air coming out from the impeller is deflected by the outlet duct in the direction from the surface.

A pressing force appears in the region (2) due to refraction

$$
F_{2}=\left(p_{0}-p_{2}\right) \pi r_{2}^{2}=\frac{2 Q^{2}}{\rho \pi r_{2}^{2}}
$$

The pressing force in the region (1) is

$$
F_{1}=\int_{r_{2}}^{r_{1}}\left[p_{0}-p_{1}(x)\right] d S
$$

Where $d S=\pi(x+d x)^{2}-\pi x^{2} \approx 2 \pi x d x$ - the elementary area over which the pressing occurs, then 


$$
F_{1}=\int_{r_{2}}^{r_{1}} \frac{Q^{2}}{8 \rho \pi^{2} x^{2} h^{2}} 2 \pi x d x=\frac{Q^{2}}{4 \pi \rho}\left(\int_{r_{2}}^{r_{1}} \frac{d x}{x h^{2}}\right)=\frac{Q^{2}}{4 \pi \rho}\left(\frac{\ln \left[r_{1} / r_{2}\right]}{h^{2}}\right)
$$

If we assume that $r_{1}=k r_{2}(k>1), r_{2}=n h(n>1)$, then the pressing force will be expressed by the following equation:

$$
F_{1}=\frac{Q^{2}}{4 \pi \rho r_{2}^{2}} n^{2} \ln k
$$

Consider the function $\frac{F_{1}}{F_{R}}=\frac{n^{2} \ln k}{4}$ (Fig.2)

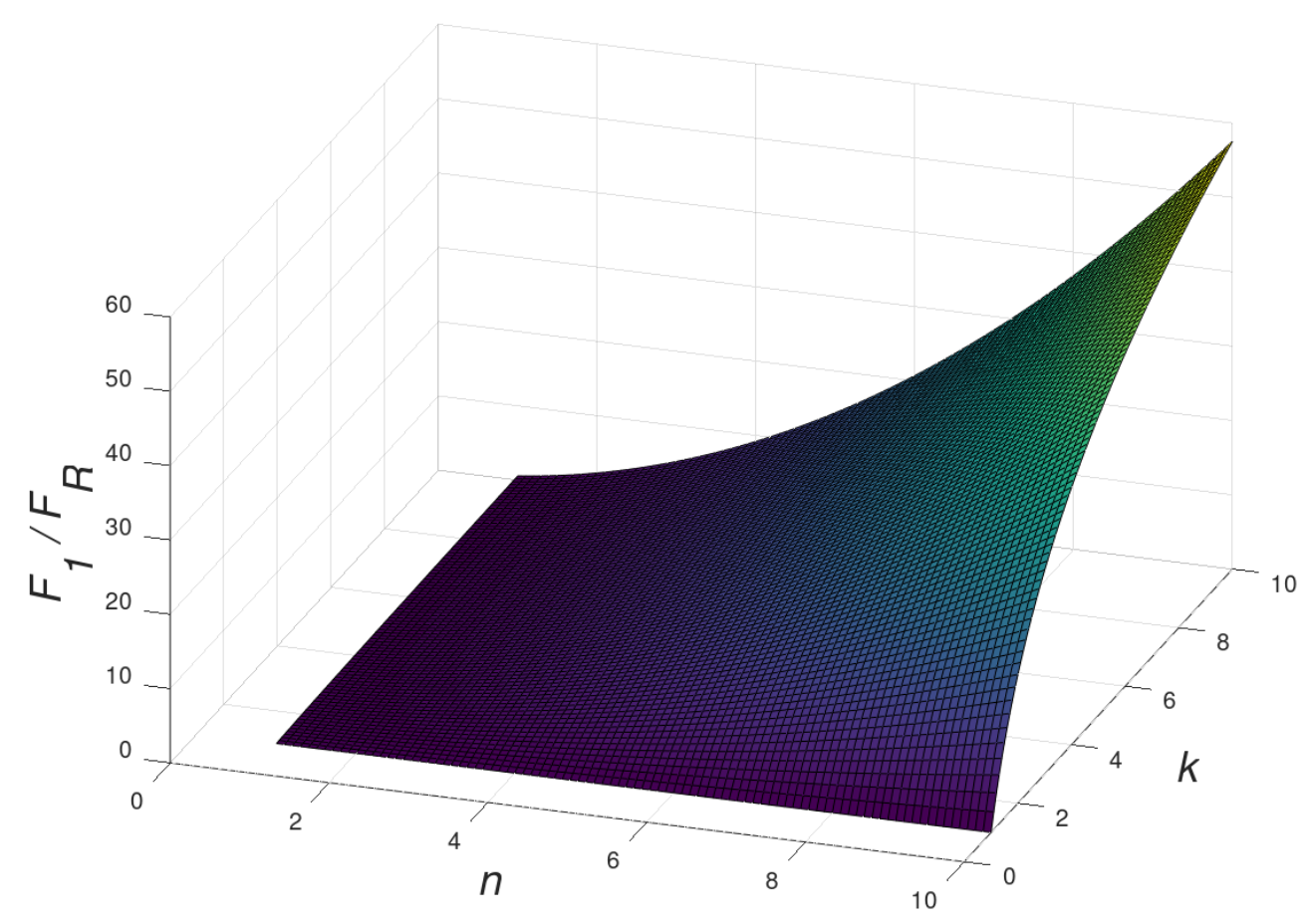

Fig. 2. Fixing force

It can be seen from the graph that the fixing force $F_{1}$ significantly exceeds the pressure due to the jet stream $F_{R}$ and due to rarefaction in the fan region $F_{2}=2 F_{R}$ even with $h$ gaps comparable to $r_{2}$.

The resulting pressure force is expressed as follows:

$$
F_{p}=F_{R}+F_{1}+F_{2}=\frac{Q^{2}}{\pi \rho}\left(\frac{3}{r_{2}^{2}}+\frac{\ln \left[\left(r_{1}-l\right) / r_{2}\right]}{4 h^{2}}\right)=K Q^{2}
$$

where $K=\frac{1}{\pi \rho}\left(\frac{3}{r_{2}^{2}}+\frac{\ln \left[\left(r_{1}-l\right) / r_{2}\right]}{4 h^{2}}\right)$ - the constant, depending on the geometry of the duct and air density. 


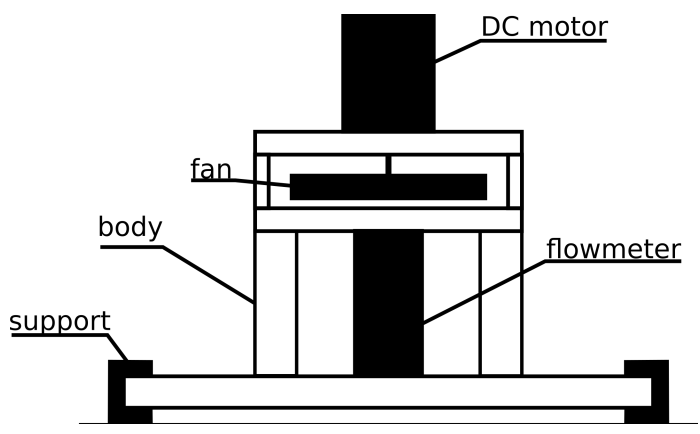

Fig. 3. Air current lines in the fixing device

\section{Experimental verification}

A prototype of an aerodynamic fixing device was created to study various parameters that affect the process of pressing to the surface. At the base of the pressing device is a flat hard disk with a hole in the center. Through this hole, the air passing through the channel with the flow meter is pumped out by means of a vacuum pump of the centrifugal type. Mass Airflow Sensors AWM720P1 is used. The impeller of the vacuum pump is driven by a DC motor. A constant gap is maintained between the disk and the fixing surface. The device body is supported on the surface by means of three supports under which pressure sensors are installed. The film pressure sensors FSP 402 were used. The supports are located at the vertices of a regular triangle, which ensures static stability of the entire structure and uniform load distribution between the force sensors. The value of the pressure force is obtained by summing the force sensor readings. An example of the data obtained for an centrifugal impeller compared with the theoretical results is shown in fig. 4 . The following parameters of the experimental prototype were taken for calculation: $r_{1}=0.25 \mathrm{~m}, r_{2}=0.019$ $\mathrm{m}, h=0.003 \mathrm{~m}, \rho=1.2 \mathrm{~kg} / \mathrm{m}^{3}$.

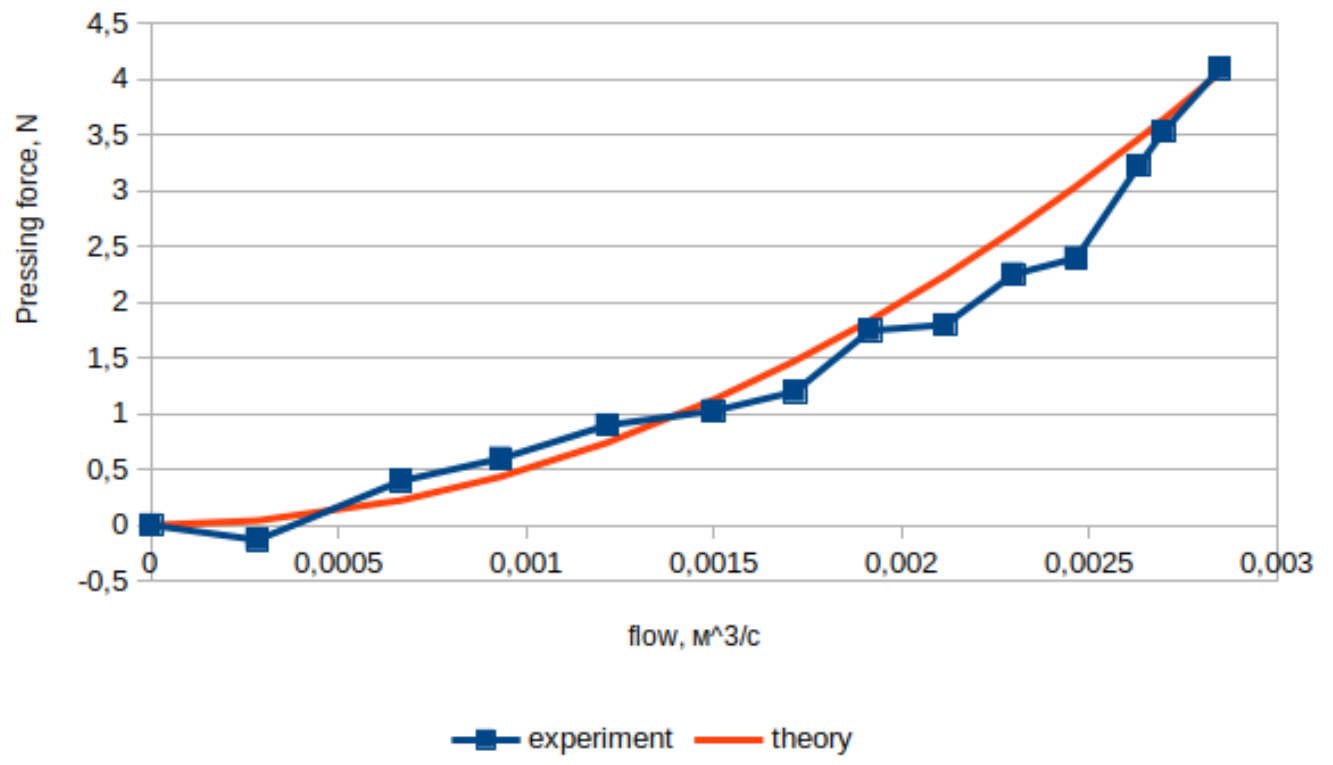

Fig. 4. Air current lines in the fixing device 


\section{Conclusion}

The dependence of the pressing force of the aerodynamic fixing device on the air flow is obtained. The influence of reactive force and rarefaction in various areas of the fixing device on the fixing force is estimated. The calculated results are compared with experimental. Experiment confirms that the fixing force is proportional to the square of the air flow rate pumped out by the fan, and the proportionality coefficient is determined only by the geometry of the pressing device and the gap between the housing and the surface.

\section{Acknowledgments}

This work was supported by RFBR grant \# 18-01-00650A

\section{References}

1. J. Xiao and A. Sadegh, City-Climber: A New Generation Wall-Climbing Robots, in Climbing and Walking Robots: towards New Applications, 10 2007, pp. 383-402.

2. P. Saboori, W. Morris, J. Xiao and A. Sadegh, Aerodynamic analysis of city-climber robots, in 2007 IEEE International Conference on Robotics and Biomimetics (ROBIO), Dec 2007.

3. N. Narayanasamy, D. R. Uppu, G. Muthukumaran, R. V and A. Singh, Procedia Computer Science 133, 847(01 2018).

4. J. Li, Xueshan Gao, Ningjun Fan, Wei Zhu, Jin Yin and Yujiao Jia, Wall climbing robot based on negative pressure-thrust suction method, in 2008 IEEE International Conference on Mechatronics and Automation, Aug 2008.

5. Q. Zhi-yuan, Z. Yan-zheng, F. Zhuang and W. Yan, International Journal of Advanced Robotic Systems 3(09 2006).

6. Xueshan Gao and K. Kikuchi, Study on a kind of wall cleaning robot, in 2004 IEEE International Conference on Robotics and Biomimetics, Aug 2004.

7. I. Koo, D. T. Tran, Y. Lee, H. Moon, J. Koo, S. Park and H. Choi, Journal of Intelligent and Robotic Systems 72(10 2013).

8. X. Li and L. Dong, IEEE/ASME Transactions on Mechatronics 21, 1024(April 2016).

9. Z. Qiang and L. Xin, Experimental investigation on climbing robot using rotation-flow adsorption unit, in Robotics and Autonomous Systems, (Elsevier, 07 2018), pp. 112-120.

10. A. Brusell, G. Andrikopoulos and G. Nikolakopoulos, Novel considerations on the negative pressure adhesion of electric ducted fans: An experimental study, in 2017 25th Mediterranean Conference on Control and Automation (MED), July 2017.

11. G. Andrikopoulos and G. Nikolakopoulos, Journal of Intelligent and Robotic Systems (08 2018).

12. J. Zhao, X. Li and J. Bai, Experimental study of vortex suction unit-based wall-climbing robot on walls with various surface conditions, in Proc IMechE Part C: J Mechanical Engineering Science (IMechE 2018), 2018.

13. G. Y. Stepanov, Hydrodynamic Theory of devices on air cushion (in russian) (Publishing House Mashgiz, Moscow, 1963). 